\title{
Investigation of a class of two-dimensional conjugate integral equation with fixed super-singular kernels
}

\author{
Nusrat Rajabov and Miklós Rontó
}




\title{
INVESTIGATION OF A CLASS OF TWO-DIMENSIONAL CONJUGATE INTEGRAL EQUATION WITH FIXED SUPER-SINGULAR KERNELS
}

\author{
NUSRAT RAJABOV AND MIKLÓS RONTÓ
}

Received December 18, 2010

\begin{abstract}
In this paper, two-dimensional linear conjugate Volterra integral equations containing super-singularities in the kernels are considered. The existence of a unique solution in a certain function class is established. Formulas representing the solution are given.
\end{abstract}

2000 Mathematics Subject Classification: 45D05

Keywords: conjugate Volterra integral equation, super-singularity in kernel, two-dimensional integral equation

\section{INTRODUCTION}

Let $D$ denote the rectangle $D=\left\{a<x<a_{0}, b_{0}<y<b\right\}$ and introduce the sets $\Gamma_{1}=\left\{a<x<a_{0}, y=b\right\}$ and $\Gamma_{2}=\left\{x=a, b_{0}<y<b\right\}$. In $D$, we consider the two-dimensional integral equation

$$
\begin{aligned}
u(x, y)+\lambda \int_{a}^{x} \frac{u(t, y)}{(t-a)^{\alpha}} d t- & \mu \int_{y}^{b} \frac{u(x, s)}{(b-s)^{\beta}} d s \\
& +\delta \int_{a}^{x} \frac{d t}{(t-a)^{\alpha}} \int_{y}^{b} \frac{u(t, s)}{(b-s)^{\beta}} d s=f(x, y),
\end{aligned}
$$

and the integral equation conjugate to equation (1.1)

$$
\begin{aligned}
T_{\lambda, \mu}^{\alpha, \beta}(v) \equiv v(x, y)+ & \frac{\lambda}{(x-a)^{\alpha}} \int_{x}^{a_{0}} v(t, y) d t-\frac{\mu}{(b-y)^{\beta}} \int_{b_{0}}^{y} v(x, s) d s \\
& +\frac{\delta}{(x-a)^{\alpha}(b-y)^{\beta}} \int_{x}^{a_{0}} d t \int_{b_{0}}^{y} v(t, s) d s=g(x, y),
\end{aligned}
$$

where $\alpha>1, \beta>1,\{\lambda, \mu, \delta\} \subset \mathbb{R}, f(x, y), g(x, y)$ are the given functions and $u(x, y), v(x, y)$, are the unknown functions. 
By the study of the solutions of the integral equations (1.1) and (1.2), the problem can be reduced to the determination of the continuous solution of a hyperbolic equation with two super-singular lines and its conjugate equation in $D$.

We seek a solution of equation (1.1) in the class of functions $u(x, y) \in C(\bar{D})$ that vanish on the singular lines $\Gamma_{1}$ and $\Gamma_{2}$. Moreover, we will assume that the unknown function $u(x, y)$ vanishes as $x \rightarrow a$ by an order higher than $\alpha-1$, and it vanishes as $y \rightarrow b$ by an order higher than $\beta-1$.

We note that the one-dimensional integral equations of types (1.1) and (1.2) are studied in [2,4-6]. Two-dimensional, three-dimensional and some many-dimensional Volterra type integral equations of type (1.1) are studied in [2,3,7-9]. One-dimensional singular integral equations with Cauchy kernels are considered in [1].

\section{THE CASE WHERE $\delta=-\lambda \mu$}

In this case, the integral equation (1.2) can be represented in the following form:

$$
\begin{aligned}
v(x, y)-\frac{\mu}{(b-y)^{\beta}} & \int_{b_{0}}^{y} v(x, s) d s \\
& +\frac{\lambda}{(x-a)^{\alpha}} \int_{x}^{a_{0}}\left[v(t, y)-\frac{\mu}{(b-y)^{\beta}} \int_{b_{0}}^{y} v(t, s) d s\right] d t=g(x, y) .
\end{aligned}
$$

If we introduce a new unknown function

$$
W(x, y)=v(x, y)-\frac{\mu}{(b-y)^{\beta}} \int_{b_{0}}^{y} v(x, s) d s,
$$

we arrive to a one-dimensional conjugate Volterra type integral equation

$$
W(x, y)+\frac{\lambda}{(x-a)^{\alpha}} \int_{x}^{a_{0}} W(t, y) d t=g(x, y) .
$$

In the case where $a<x<a_{0}$, according to [3], the integral equation (2.2) has a unique solution which is given by the formula

$$
W(x, y)=g(x, y)-\frac{\lambda}{(x-a)^{\alpha}} \int_{x}^{a_{0}} \exp \left[\lambda\left(\omega_{a}^{\alpha}(t)-\omega_{a}^{\alpha}(x)\right)\right] g(t, y) d t,
$$

where $\omega_{a}^{\alpha}(x)=\left[(\alpha-1)(x-a)^{\alpha-1}\right]^{-1}$.

Analogously, the solution of the integral equation (2.1), for $b_{0}<y<b$, is given by the formula

$$
v(x, y)=W(x, y)+\frac{\mu}{(b-y)^{\beta}} \int_{b_{0}}^{y} \exp \left[\mu\left(\omega_{b}^{\beta}(y)-\omega_{b}^{\beta}(s)\right)\right] W(t, y) d s,
$$

where $\omega_{b}^{\beta}(y)=\left[(\beta-1)(b-y)^{\beta-1}\right]^{-1}$.

By substituting the value of $W(x, y)$ into (2.4), we obtain a general solution of the integral equation (1.2) in the form 


$$
\begin{aligned}
v(x, y)=g(x, y) & -\frac{\lambda}{(x-a)^{\alpha}} \int_{x}^{a_{0}} \exp \left[\lambda\left(\omega_{a}^{\alpha}(t)-\omega_{a}^{\alpha}(x)\right)\right] g(t, y) d t \\
& +\frac{\mu}{(b-y)^{\beta}} \int_{b_{0}}^{y} \exp \left[\mu\left(\omega_{b}^{\beta}(y)-\omega_{b}^{\beta}(s)\right)\right] g(x, s) d s \\
& -\frac{\lambda \mu}{(x-a)^{\alpha}(b-y)^{\beta}} \int_{x}^{a_{0}} \exp \left[\lambda\left(\omega_{a}^{\alpha}(t)-\omega_{a}^{\alpha}(x)\right)\right] d t \\
& \times \int_{b_{0}}^{y} \exp \left[\mu\left(\omega_{b}^{\beta}(y)-\omega_{b}^{\beta}(s)\right)\right] g(t, s) d s \equiv\left(T_{\lambda, \mu}^{\alpha, \beta}\right)^{-1} g .
\end{aligned}
$$

We thus obtain the following

Theorem 1. Assume that in equation (1.2) the parameters are related by the equality $\delta=-\lambda \mu$, and $g(x, y) \in C(D)$. Then the non-homogeneous integral equation (1.2) has a unique solution in class $C(D)$, which is given by formula (2.5).

\section{THE CASE Where $\delta \neq-\lambda \mu$}

In this case, the integral equation (1.2) can be represented in the following form:

$$
\begin{aligned}
& T_{\lambda, \mu}^{\alpha, \beta}(v) \equiv v(x, y)+\frac{\lambda}{(x-a)^{\alpha}} \int_{x}^{a_{0}} v(t, y) d t \\
&-\frac{\mu}{(b-y)^{\beta}} \int_{b_{0}}^{y} v(x, s) d s-\frac{\lambda \mu}{(x-a)^{\alpha}(b-y)^{\beta}} \int_{x}^{a_{0}} d t \int_{b_{0}}^{y} v(t, s) d s \\
& \quad=g(x, y)-\frac{\delta_{1}}{(x-a)^{\alpha}(b-y)^{\beta}} \int_{x}^{a_{0}} d t \int_{b_{0}}^{y} v(t, s) d s .
\end{aligned}
$$

Let us introduce the function

$$
g_{1}(x, y)=g(x, y)-\frac{\delta_{1}}{(x-a)^{\alpha}(b-y)^{\beta}} \int_{x}^{a_{0}} d t \int_{b_{0}}^{y} v(t, s) d s,
$$

where $\delta_{1}=\delta+\lambda \mu$. Clearly, $g_{1}(x, y) \in C(D)$. Then the solution of the integral equation $T_{\lambda, \mu}^{\alpha, \beta}(v)=g_{1}(x, y)$ is as follows:

$$
\begin{aligned}
v(x, y)=g_{1}(x, y)-\frac{\lambda}{(x-a)^{\alpha}} \int_{x}^{a_{0}} \exp \left[\lambda\left(\omega_{a}^{\alpha}(t)-\omega_{a}^{\alpha}(x)\right)\right] g_{1}(t, y) d t \\
\quad+\frac{\mu}{(b-y)^{\beta}} \int_{b_{0}}^{y} \exp \left[\mu\left(\omega_{b}^{\beta}(y)-\omega_{b}^{\beta}(s)\right)\right] g_{1}(x, s) d s \\
\quad-\frac{\lambda \mu}{(x-a)^{\alpha}(b-y)^{\beta}} \int_{x}^{a_{0}} \exp \left[\lambda\left(\omega_{a}^{\alpha}(t)-\omega_{a}^{\alpha}(x)\right)\right] d t \\
\quad \times \int_{b_{0}}^{y} \exp \left[\mu\left(\omega_{b}^{\beta}(y)-\omega_{b}^{\beta}(s)\right)\right] g_{1}(t, s) d s \equiv\left(T_{\lambda, \mu}^{\alpha, \beta}\right)^{-1} g_{1}(x, y) .
\end{aligned}
$$


In formula (3.1), by substituting the value of $g_{1}(x, y)$ and rearranging the appropriate terms, we arrive to the solution of the integral equation

$$
\Phi(x, y)+\frac{\delta_{1}}{(x-a)^{\alpha}(b-y)^{\beta}} \int_{x}^{a_{0}} d t \int_{b_{0}}^{y} \Phi(t, s) d s=E_{\lambda, \mu}^{\alpha, \beta}[g(x, y)],
$$

where

$$
\begin{gathered}
E_{\lambda, \mu}^{\alpha, \beta}[g(x, y)]=\exp \left[\lambda \omega_{a}^{\alpha}(x)-\mu \omega_{b}^{\beta}(y)\right]\left(T_{\lambda, \mu}^{\alpha, \beta}\right)^{-1} g(x, y) \\
=\exp \left[\lambda \omega_{a}^{\alpha}(x)-\mu \omega_{b}^{\beta}(y)\right] g(x, y) \\
-\frac{\lambda}{(x-a)^{\alpha}} \exp \left[-\mu \omega_{b}^{\beta}(y)\right] \int_{x}^{a_{0}} \exp \left[\lambda \omega_{a}^{\alpha}(t)\right] g(t, y) d t \\
+\frac{\mu}{(b-y)^{\beta}} \exp \left[\lambda \omega_{a}^{\alpha}(x)\right] \int_{b_{0}}^{y} \exp \left[-\mu \omega_{b}^{\beta}(s)\right] g(x, s) d s \\
-\frac{\lambda \mu}{(x-a)^{\alpha}(b-y)^{\beta}} \int_{x}^{a_{0}} \exp \left[\lambda \omega_{a}^{\alpha}(t)\right] d t \int_{b_{0}}^{y} \exp \left[-\mu \omega_{b}^{\beta}(s)\right] g(t, s) d s
\end{gathered}
$$

and

$$
\Phi(x, y)=\exp \left[\lambda \omega_{a}^{\alpha}(x)-\mu \omega_{a}^{\alpha}(y)\right] v(x, y) .
$$

4. REPRESENTATION OF A SOLUTION BY FUNCTIONAL SERIES OF $\exp \left(-\omega_{a}^{\alpha}(x)\right)$

We seek a solution for integral equation (3.2) in the class of functions that can be represented in the form

$$
\Phi(x, y)=\sum_{n=1}^{\infty}\left(\exp \left(-\omega_{a}^{\alpha}(x)\right)\right)^{n} \Phi_{n}(y)(x-a)^{-\alpha},
$$

where $\Phi_{n}(y)$ are unknown functions.

We assume that function $g(x, y)$ admits representation in the form

$$
g(x, y)=\exp \left[-\lambda \omega_{a}^{\alpha}(x)+\mu \omega_{b}^{\beta}(y)\right] \sum_{n=1}^{\infty}\left[\exp \left(-\omega_{a}^{\alpha}(x)\right)^{n}\right](x-a)^{-\alpha} g_{n}(y),
$$

where $g_{n}(y)$ are known functions. Moreover, assume that the series (4.2) converges absolutely and uniformly. By substituting this value $g(x, y)$ into (3.3), we have

$$
\begin{aligned}
E_{\lambda, \mu}^{\alpha, \beta}[g(x, y)]= & \sum_{n=1}^{\infty}\left[\exp \left(-\omega_{a}^{\alpha}(x)\right)\right]^{n}(x-a)^{-\alpha} \\
& \times\left(\frac{n+\lambda}{n}\right)\left[g_{n}(y)+\mu(b-y)^{-\mu} \int_{b_{0}}^{y} g_{n}(s) d s\right] \\
-(x-a)^{-\alpha} \lambda & {\left[\sum_{n=1}^{\infty} n^{-1} \exp \left(-\omega_{a}^{\alpha}\left(a_{0}\right)\right)^{n}\left(g_{n}(y)+\mu(b-y)^{-\mu} \int_{b_{0}}^{y} g_{n}(s) d s\right)\right] . }
\end{aligned}
$$


By substituting the values of $\Phi(x, y)$ and $E_{\lambda, \mu}^{\alpha, \beta}[g(x, y)]$ into the integral equation (3.2) and equating the coefficients at $\left[\exp \left(-\omega_{a}^{\alpha}(x)\right)\right]^{k}, k=0,1,2, \ldots$, we obtain the following relations between the functions $\Phi_{n}(y)$ and $g_{n}(y), n=0,1,2, \ldots$ :

$$
\begin{aligned}
\delta_{1}(b-y)^{-\beta} & \sum_{n=1}^{\infty} n^{-1}\left[\exp \left(-\omega_{a}^{\alpha}\left(a_{0}\right)\right)\right]^{n} \int_{b_{0}}^{y} \Phi_{n}(s) d s \\
& =-\sum_{n=1}^{\infty} n^{-1}\left[\exp \left(-\omega_{a}^{\alpha}\left(a_{0}\right)\right)\right]^{n} \lambda\left[g_{n}(y)+(b-y)^{-\beta} \mu \int_{b_{0}}^{y} g_{n}(s) d s\right]
\end{aligned}
$$

and

$$
\Phi_{n}(y)-\frac{\delta_{1}}{n(b-y)^{\beta}} \int_{b_{0}}^{y} \Phi_{n}(s) d s=\left(\frac{n+\lambda}{n}\right) g_{n}(y)+\frac{\mu(n+\lambda)}{n(b-y)^{\mu}} \int_{b_{0}}^{y} g_{n}(s) d s .
$$

According to [3], if the system of integral equation (4.4) has a solution, then it can be represented in the form

$$
\begin{aligned}
\Phi_{n}(y)= & \frac{n+\lambda}{n}\left[g_{n}(y)\right. \\
& \left.+\left(\frac{\mu n-\delta_{1}}{n}\right) \frac{1}{(b-y)^{\beta}} \int_{b_{0}}^{y} \exp \left[\frac{\delta_{1}}{n}\left(\omega_{b}^{\beta}(s)-\omega_{b}^{\beta}(y)\right)\right] g_{n}(s) d s\right],
\end{aligned}
$$

where $n=0,1,2, \ldots, \omega_{b}^{\beta}(y)=\left[(\beta-1)(b-y)^{\beta-1}\right]^{-1}$.

Furthermore, it follows from equality (4.3) that

$$
\delta_{1}(b-y)^{-\beta} \int_{b_{0}}^{y} \Phi_{n}(s) d s=-\lambda\left[g_{n}(y)+\mu(b-y)^{-\beta} \int_{b_{0}}^{y} g_{n}(s) d s\right]
$$

for $n=0,1,2, \ldots$. From expression (4.6), by substituting the values of $\Phi_{n}(s)$ according to formula (4.5), we obtain the equality

$$
\begin{aligned}
\left(\frac{n+\lambda}{n}\right)\left[\frac{\mu n}{(b-y)^{\beta}} \int_{b_{0}}^{y} g_{n}(s) d s\right. & \\
& \left.-\frac{\left(\mu n-\delta_{1}\right)}{(b-y)^{\beta}} \int_{b_{0}}^{y} \exp \left(\frac{\delta_{1}}{n}\left(\omega_{b}^{\beta}(s)-\omega_{b}^{\beta}(y)\right)\right) g_{n}(s) d s\right] \\
& =-\lambda\left[g_{n}(y)+\frac{\mu}{(b-y)^{\beta}} \int_{b_{0}}^{y} g_{n}(s) d s\right], \quad n=0,1,2,3 \ldots
\end{aligned}
$$

From formula (4.1), by substituting the value $\Phi_{n}(y)$ from equality (4.5), where $\Phi(x, y)=v(x, y) \exp \left[\lambda \omega_{a}^{\alpha}(x)-\mu \omega_{b}^{\beta}(y)\right]$, we find 


$$
\begin{aligned}
v(x, y)= & \exp \left[-\lambda \omega_{a}^{\alpha}(x)+\mu \omega_{b}^{\beta}(y)\right] \sum_{n=1}^{\infty} \frac{\exp \left(-\omega_{a}^{\alpha}(x)\right)^{n}}{(x-a)^{\alpha}}\left(\frac{n+\lambda}{n}\right) \\
\times & {\left[g_{n}(y)+\frac{\mu n-\delta_{1}}{n(b-y)^{\beta}} \int_{b_{0}}^{y}\left(\exp \left(\omega_{b}^{\beta}(s)-\omega_{b}^{\beta}(y)\right)\right)^{\frac{\delta_{1}}{n}} g_{n}(s) d s\right] . }
\end{aligned}
$$

Thus, we arrive at the following conclusion.

Theorem 2. Assume that in the integral equation (1.2) $\delta \neq-\lambda \mu$, and that the function $g(x, y)$ is represented by series (4.2), which converges absolutely and uniformly. Then the integral equation (1.2) has a solution in the class of functions $v(x, y)$ that are representable in the form

$$
v(x, y)=\exp \left[-\lambda \omega_{a}^{\alpha}(x)+\mu \omega_{b}^{\beta}(y)\right] \sum_{n=1}^{\infty} \frac{\Phi_{n}(y)}{(x-a)^{\alpha} \exp \left(\omega_{a}^{\alpha}(x)\right)^{n}} .
$$

Moreover, if the functions $g_{k}(y), k=1,2, \ldots$, in (4.2) satisfy the infinite system of solvability conditions (4.7), then that solution is unique and can be represented by by formula (4.8).

Remark 1. In the case where $\delta \neq-\lambda \mu$, the solution of the integral equation (3.2) could be sought in the class of functions that are representable by a functional series of $\exp \left(-\omega_{b}^{\beta}(y)\right)$, i. e.,

$$
\Phi(x, y)=\sum_{n=1}^{\infty} \exp \left(-n \omega_{b}^{\beta}(y)\right)(b-y)^{-\beta} W_{n}(x)
$$

where $W_{n}(x)$ are unknown functions. Then one assumes that the function $g(x, y)$ is represented in the form

$$
g(x, y)=\exp \left[-\lambda \omega_{a}^{\alpha}(x)+\mu \omega_{b}^{\beta}(y)\right] \sum_{n=1}^{\infty} \frac{\exp \left(-n \omega_{b}^{\beta}(y)\right)}{(b-y)^{\beta}} g_{n}(x) .
$$

By modifying suitably the argument above, in that case, one can also obtain a statement similar to Theorem 2.

\section{REMARKS ON A NON-MODEL INTEGRAL EQUATION}

In the domain $D$, we consider the two-dimensional integral equation

$$
\begin{aligned}
u(x, y)+\int_{a}^{x} \frac{A(t) u(t, y)}{(t-a)^{\alpha}} d t & -\int_{y}^{b} \frac{B(s) u(x, s)}{(b-s)^{\beta}} d s \\
+ & \int_{a}^{x} \frac{d t}{(t-a)^{\alpha}} \int_{y}^{b} \frac{c(t, s) u(t, s)}{(b-s)^{\beta}} d s=f(x, y),
\end{aligned}
$$

and its conjugate equation 


$$
\begin{aligned}
v(x, y)+\frac{A(x)}{(x-a)^{\alpha}} \int_{x}^{a_{0}} v(t, y) d t-\frac{B(y)}{(b-y)^{\beta}} \int_{b_{0}}^{y} v(x, s) d s \\
\quad+\frac{c(x, y)}{(x-a)^{\alpha}(b-y)^{\beta}} \int_{x}^{a_{0}} d t \int_{b_{0}}^{y} v(t, s) d s=g(x, y) .
\end{aligned}
$$

Integral equations of form (5.1) are studied in [8].

Remark 2. One can find a solution of the integral equation (5.2) if $c(x, y) \equiv$ $-A(x) B(y)$. In that case, as is shown in [6], the question is reduced to finding a solution of two split systems of one-dimensional conjugate integral equations of type (5.2).

Remark 3. In the case where $c(x, y) \not \equiv-A(x) B(y)$, the problem of finding solution for integral equation (5.2) is reduced to the problem of the determination of a solution of the integral equation

$$
\begin{array}{r}
v(x, y) \\
+\frac{c_{1}(x, y)}{(x-a)^{\alpha}(b-y)^{\beta}} \int_{x}^{a_{0}} \exp \left[A(a)\left(\omega_{\alpha}(t)-\omega_{\alpha}(x)\right)-W_{A, \alpha}^{-}(t)-W_{A, \alpha}^{-}(x)\right] d t \\
\times \int_{b_{0}}^{y} \exp \left[B(b)\left(\omega_{b}^{\beta}(y)-\omega_{b}^{\beta}(s)\right)+W_{b, \beta}^{-}(s)-W_{b, \beta}^{-}(y)\right] v(t, s) d s \\
\equiv\left(T_{A(x), B(y)}^{\alpha, \beta}\right)^{-1}(g(x, y)),
\end{array}
$$

for any $(x, y) \in D$, where $\left(T_{A(x), B(y)}^{\alpha, \beta}\right)^{-1}$ is a known integral operator.

\section{ACKNOWLEDGEMENT}

This research was carried out as part of the TAMOP-4.2.1.B-10/2/KONV-20100001 project with support by the European Union, co-financed by the European Social Fund.

\section{REFERENCES}

[1] F. D. Gakhov, Boundary value problems, I. N. Sneddon, Ed. Oxford-New York-Paris; Reading, Mass.-London: Pergamon Press; Addison-Wesley Publishing Co., Inc., 1966.

[2] R. N., Volterra type integral equations with boundary and interior fixed singular and supersingular kernels and their application. Dushanbe: Tehran University, 2010.

[3] N. Rajabov, An introduction to the theory of partial differential equations with super-singular coefficients. Dushanbe: Tehran University, 1992.

[4] N. Rajabov, “On a Volterra integral equation,” Dokl. Math., vol. 65, no. 2, pp. 217-220, 2002.

[5] N. Rajabov, "About one class of Volterra type linear integral equations with an interior fixed singular or super-singular point," in Topics in analysis and its applications, ser. NATO Sci. Ser. II Math. Phys. Chem., vol. 147. Dordrecht: Kluwer Acad. Publ., 2004, pp. 317-326.

[6] N. Rajabov, Volterra type integral equation with fixed boundary and interior singular and supersingular kernels. Dushanbe: "Devashtich" Publishers, 2007. 
[7] N. Rajabov, "About one class of second-order linear hyperbolic equations for which all of the boundary consist of singular lines," in Further progress in analysis. Hackensack, NJ: World Sci. Publ., 2009, pp. 356-367.

[8] N. Rajabov and L. Rajabova, "On the theory of a class of two-dimensional, non-model integral Volterra type equations with boundary supersingularities in the kernels," Doklady Mathematics, vol. 71, no. 1, pp. 111-114, 2005.

[9] N. Rajabov, M. Rontó, and L. Rajabova, "On some two dimensional Volterra type linear integral equations with super-singularity,” Math. Notes (Miskolc), vol. 4, no. 1, pp. 67-76, 2003.

Authors' addresses

Nusrat Rajabov

Tajik National University, 17 Rudaki Ave., 734025 Dushanbe, Tajikistan

E-mail address: nusrat38@mail.ru

Miklós Rontó

Institute of Mathematics, University of Miskolc, H-3515 Miskolc-Egyetemváros, Hungary

E-mail address: matrontodgold.uni-miskolc.hu 